02,12

\title{
Локальные и интегральные свойства квазиодномерного сверхпроводника в режиме квантовых флуктуаций параметра порядка
}

\author{
(C) К.Ю. Арутюнов ${ }^{1,2}$, Я.С. Лехтинен ${ }^{3,4}$, Д.О. Трефилов ${ }^{1}$, А.А. Радкевич ${ }^{5}$, А.Г. Семенов ${ }^{1,5}$, А.Д. Заикин ${ }^{5,6}$ \\ ${ }^{1}$ Национальный исследовательский университет „Высшая школа экономики“, \\ Москва, Россия \\ ${ }^{2}$ Институт фоизических проблем им. П.Л. Капицы РАН, \\ Москва, Россия \\ ${ }^{3}$ VTT Technical Research Centre of Finland Ltd., \\ 02150 Espoo, Finland \\ ${ }^{4}$ Department of Physics, University of Jyvaskyla, \\ PB 35, Fl-40014 Jyvaskyla, Finland \\ ${ }^{5}$ Физический институт РАН им. П.Н. Лебедева, \\ Москва, Россия \\ ${ }^{6}$ Institute for Quantum Materials and Technologies, Karlsruhe Institute of Technology (KIT), \\ 76021 Karlsruhe, Germany \\ E-mail: karutyunov@hse.ru
}

Поступила в Редакцию 9 апреля 2021 г.

В окончательной редакции 9 апреля 2021 г.

Принята к публикации 19 апреля 2021 г.

Использование сверхпроводящих материалов для наноэлектронных устройств нового поколения представляется крайне заманчивым с точки зрения отсутствия диссипации энергии при протекании электрического тока. Однако в системах малых размеров роль флуктуаций может быть весьма существенной. В работе экспериментально и теоретически исследовались транспортные свойства тонких сверхпроводящих нанополосок из титана. Удалось показать, что квантовые флуктуации параметра порядка различным образом влияют на интегральные и локальные характеристики квазиодномерного сверхпроводника. В достаточно тонких нанопроводах при самых низких температурах может наблюдаться конечное электрическое сопротивление, тогда как туннельные вольт-амперные характеристики обнаруживают лишь слегка размытые щелевые особенности и конечный джозефсоновский ток. Явление имеет принципиальное значение для объяснения феномена мезоскопической сверхпроводимости и должно учитываться при проектировании криоэлектронных устройств нанометровых масштабов.

Ключевые слова: сверхпроводимость, квантовые флуктуации, туннельные контакты.

DOI: 10.21883/FTT.2021.09.51244.33H

\section{1. Введение}

В последние годы наблюдается замедление роста степени интеграции коммерческих микро- и наноэлектронных устройств, формально знаменующее нарушение закона Мура [1]. Тому можно привести две причины: большое тепловыделение на единицу объема (или площади) и различные квантовые размерные эффекты. Радикальным решением первой проблемы может быть переход в критических элементах наноэлектронных схем от нормальных металлов или полупроводников к сверхпроводящим материалам. В сверхпроводниках мезоскопических размеров наблюдается ряд эффектов, которые могут привести к качественно новым применениям: например, элементам квантовой логики (кубитам) [2] и квантовому эталону силы электрического поля [3,4].

Хорошо известно, что сверхпроводимость - макроскопическое квантовое явление, описываемое комплексным параметром порядка $\Delta=|\Delta| e^{i \phi}$. Основное состоя- ние нетоконесущего массивного сверхпроводника характеризуется одинаковым значением фазы $\phi$ и модуля $|\Delta|$ во всем объеме. Однако с уменьшением размерности системы флуктуации начинают играть важную роль. В случае квазиодномерного канала флуктуации параметра порядка могут существенно повлиять на его физические свойства $[5,6]$. Достаточно близко к критической температуре $T \rightarrow T_{c}$ преобладающий вклад вносят тепловые флуктуации, тогда как квантовые флуктуации [7] даже при $T \ll T_{c}$ приводят к конечному сопротивлению нанопроводов [8-10] и подавлению мейсснеровских токов в замкнутом сверхпроводящем контуре [11,12].

Перечисленные феноменологические атрибуты сверхпроводимости, нулевое сопротивление и диамагнетизм, отражают интегральные свойства системы. С появлением микроскопической модели Бардина-КупераШриффера (БКШ) стало также понятным, что сверхпроводящее состояние в „классических“ сверхпроводящих материалах неразрывно связано с наличием щели 
в спектре квазичастичных возбуждений. Энергетическая щель является локальным параметром сверхпроводника, который может быть измерен „в точке“ методом туннельной спектроскопии, в отличие, например, от электронной проводимости, требующей образцов достаточной протяженности. Целью настоящей работы является сравнительный анализ влияния квантовых флуктуаций параметра порядка на локальные и интегральные свойства квазиодномерных сверхпроводящих каналов.

\section{2. Образцы и методика эксперимента}

Методом взрывной электронной литографии и направленного вакуумного напыления были изготовлены гибридные наноструктуры, представляющие собой длинную алюминиевую полоску с шириной линии порядка $150 \mathrm{~nm}$ и толщиной $30 \mathrm{~nm}$, окисленную в атмосфере чистого кислорода с последующим напылением поперечных титановых нанопроводов (рис. 1, a). Сечение алюминиевого электрода выбиралось существенно больше того значения, когда в этом материале необходимо учитывать флуктуационные явления $[9,13]$. А вот сечение титановых нанопроводов (длина $X=20 \mu \mathrm{m}$ ) выбиралось так, чтоб перекрыть область от сильного до слабого вклада квантовых флуктуаций параметра порядка. Учитывая экспоненциальную зависимость частоты квантовых проскальзываний фазы (КПФ) от сечения сверхпроводящего канала [5,7], в наших предыдущих работах $[10,11,14,15]$ было показано, что для толщины титановой полоски $d=30 \mathrm{~nm}$ вариации ширины $w$ от 30 до $60 \mathrm{~nm}$ как раз соответствуют обозначенному диапазону. Следует также

$a$
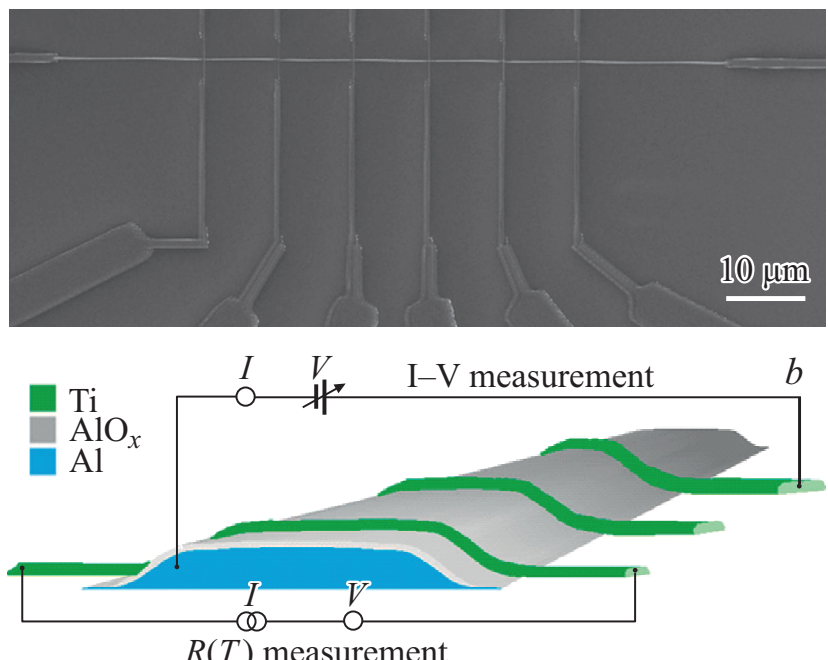

Рис. 1. a) Микрофотография типичной наноструктуры. Горизонтальная линия - алюминиевая (Al) полоска. Вертикальные линии - титановые (Тi) электроды различной ширины, перекрывающие алюминий через тонкий слой окиси алюминия $\left(\mathrm{AlO}_{x}\right)$, формируя туннельные контакты. $b$ ) Схематика наноструктуры и измерений. заметить, что для наших титановых нанопроводов сверхпроводящая длина когерентности $\xi(T \rightarrow 0) \approx 140 \mathrm{~nm}$, что свидетельствует о выполнении условия квазиодномерности $d, w \ll \xi \ll X$ в широком температурном диапазоне не слишком близко от $T_{c}$.

На одной и той же многотерминальной структуре (рис. $1, b)$ можно было измерять как вольт-амперные $V(I, T=\mathrm{const})$ и вольт-температурные $V(T, I=\mathrm{const})$ зависимости отдельных титановых нанопроводов, так и - туннельные $V(I)$ характеристики в точках перекрытия алюминиевых и титановых нанополосок $\left(\mathrm{Al}-\mathrm{AlO}_{x}-\mathrm{Ti}\right.$ junction). Все измерения проводились в рефрижераторе растворения $3 \mathrm{He} 4 \mathrm{He}$ с использованием аналоговых предусилителей напряжения и тока, связывающих криогенную цепь с комнатной цифровой электроникой. Особое внимание уделялось фильтрации входных и выходных линий от внешних электромагнитных наводок [16]. Производные вольт-амперных характеристик (BAX) $d I / d V(V)$ измерялись модуляционным методом с использованием фазочувствительного детектирования. Экспериментальные данные собирались и обрабатывались автоматизированной установкой на базе персонального компьютера.

\section{3. Результаты и обсуждение}

Типичные зависимости сопротивления от температуры $R(T)$ для двух титановых нанопроводов с различной шириной линии w приведены на рис. 2. В силу технических ограничений изготовления наноструктур (минимизация контактных площадок на чипе), измерения $R(T)$ были возможны только в псевдо-4-контактной конфигурации (рис. 2, вставка). Хорошо видно, что сверхпроводящий переход более тонкой нанополоски $(w=40 \mathrm{~nm})$ существенно более уширен по сравнению с широким образцом $(w=53 \mathrm{~nm})$, и соответствующая зависимость $R(T)$ не экстраполируется к нулевому значению сопротивления при $T \rightarrow 0$. Обнаруженная тенденция типична для сверхтонких сверхпроводящих каналов, объясняется эффектом КПФ [5] и, применительно к титану [10,11,15], находится в разумном согласии с моделью [7]. Однако следует заметить, что модель [7] рассматривает КПФ как малое возмущение, т. е. система находится в сверхпроводящем состоянии, а квантовые флуктуации - редкое явление, которое, в соответствии с соотношением Джозефсона, приводит к возникновению малого усредненного по времени напряжения $\langle V\rangle=(h / 2 e) d \phi / d t$, что в режиме задания тока $I$ экспериментально проявляется как конечное сопротивление $R \cong\langle V\rangle / I$. Строго говоря, модель [7] может объяснить слабую температурную зависимость $R(T)$, как, например, для образца с $w=40 \mathrm{~nm}$ на рис. 2, только качественно: КПФ происходят так часто, что полностью „забивают“ сверхпроводящее состояние. Более углубленное понимание физики в режиме сильных флуктуаций появилось относительно недавно [17]. 


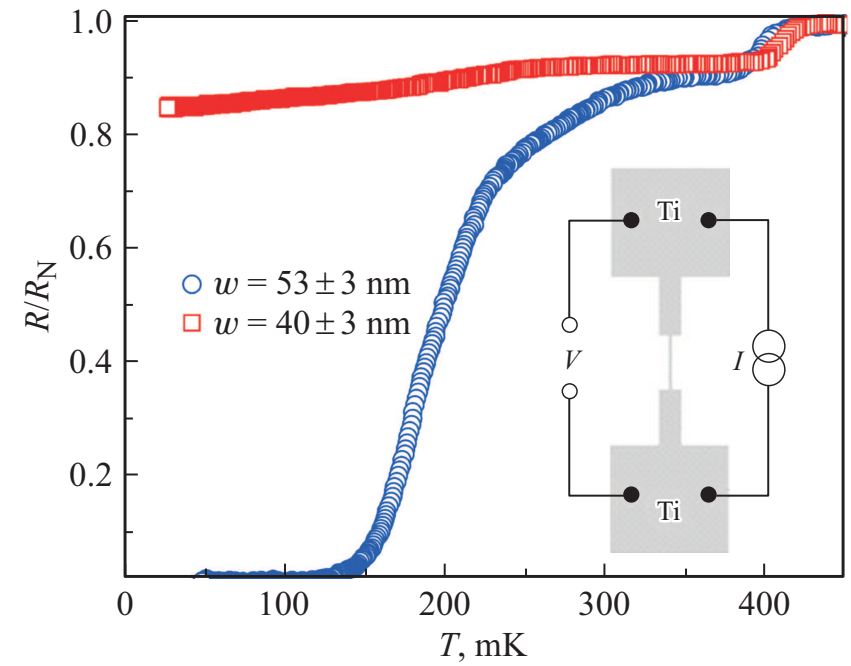

Pис. 2. Типичная зависимость сопротивления от температуры $R(T)$, нормированного на величину сопротивления в нормальном состоянии $R_{N}$, для двух титановых нанопроводов одинаковой длины $X=20 \mu \mathrm{m}$ и толщины $d=35 \mathrm{~nm}$, но разной ширины $w$, обозначенной на рисунке. Для ясности на вставке не показан алюминиевый электрод, по которому в этом эксперименте не течет электрический ток.

Квантовые флуктуации параметра порядка в квазиодномерных сверхпроводящих каналах характеризуются двумя безразмерными параметрами [17]:

$$
g_{\xi}=R_{q} / R_{\xi} \sim \sigma, \quad g_{Z}=R_{q} / Z \sim \sqrt{\sigma},
$$

где $R_{q}=h / e^{2}=25.8 \mathrm{k} \Omega$ - квант сопротивления; $R_{\xi}-$ сопротивление участка образца с длиной, равной сверхпроводящей длине когерентности $\xi ; Z=(L C)^{1 / 2}-$ импеданс; $L$ и $C-$ кинетическая индуктивность и удельная емкость образца на единицу длины, соответственно; $\sigma \approx d w$ - эффективное сечение нанопровода. Параметр $g_{\xi}$ определяет амплитуду флуктуаций модуля параметра порядка $\delta \Delta / \Delta \sim 1 / g_{\xi}-$ и квантовую амплитуду КПФ на единицу длины $\Gamma_{\mathrm{QPS}}=b\left(g_{\xi} \Delta / \xi\right) \exp \left\{-a g_{\xi}\right\}$, где $a \approx 1$ и $b \approx 1$ - численные безразмерные константы модели [18]. Параметр $g_{Z}$ описывает длинномасштабные флуктуации фазы сверхпроводящего параметра порядка за счет взаимодействия между отдельными актами КПФ через плазмонные колебания зарядовой плотности (волны Муи-Шена) [19], распространяющиеся вдоль сверхпроводника со скоростью $v \sim 1 /(L C)^{1 / 2}$. Иными словами, параметр $g_{\xi}$ ответственен за возбуждение отдельных, изолированных КПФ, тогда как $g_{Z}-$ за взаимодействие КПФ. Было показано [7], что при достаточно низкой температуре $T \rightarrow 0$ ситуация $g_{Z}=16$ соответствует переходу сверхпроводник-изолятор, разделяя „сверхпроводящее“ состояние с $g_{Z}>16$ от ,диэлектрического“ с $g_{Z}<16$. Оценки дают $3<g_{\xi}<40$ и $1<g_{Z}<3$ для исследованных в настоящей работе самых узких и самых широких титановых нанополосок, соответственно. Применительно ко всем нашим образцам, находящимся в диэлектрическом пределе, т.е. с $g_{Z}<16$ можно определить некую корреляционную длину [20,21]:

$$
X_{c} \sim \xi \exp \left\{\frac{a g_{\xi}-\ln b}{2-g_{Z} / 8}\right\},
$$

которая физически соответствует характерному масштабу локализации куперовских пар [12] за счет туннелирования квантов магнитного потока - флуксонов. Системы с длиной $X \leq X_{c}$ могут демонстрировать сверхпроводящие свойства в присутствии КПФ, тогда как в пределе $X \gg X_{c}$ сверхток полностью подавлен квантовыми флуктуациями и квазиодномерный сверхпроводник обнаруживает конечное сопротивление даже при $T \rightarrow 0$. Именно это и наблюдается в наших $R(T)$-экспериментах на нанопроводах различных сечений (рис. 2). Для образца с $w=53 \mathrm{~nm}$ оценка дает корреляционную длину $X_{c} \approx 12 \mu \mathrm{m}$, сравнимую с геометрической длиной $X=20 \mu \mathrm{m}$, и, соответственно, система остается в сверхпроводящем состоянии (с нулевым сопротивлением) при достаточно низкой температуре $T<T_{c}$, в то время как более узкий образец с $w=40 \mathrm{~nm}$, с учетом сильной экспоненциальной зависимости (2), соответствует пределу $X \gg X_{c}$ и, как следствие, даже при самых низких температурах обнаруживает конечное сопротивление (рис. 2). Идентичные зависимости были обнаружены на большом количестве титановых нанополосок и не представлены на рис. 2 исключительно ради ясности графического представления данных. Все сказанное выше о проявлении „сверхпроводящих“ свойств относится к такой интегральной характеристике системы, как электрическое сопротивление.

Разумно задаться вопросом: справедливо ли обсуждаемое флуктуационное подавление сверхпроводимости для таких локальных характеристик, как энергетическая щель и/или плотность состояний? С целью проверить эту гипотезу, на одних и тех же мультитерминальных наноструктурах измерялись ВАХ туннельных контактов $\mathrm{Al}-\mathrm{AlO}_{x}-\mathrm{Ti}$, образованных в точках перекрытия титановых и алюминиевых нанополосок через тонкий оксидный слой (рис. 1). Соответствующие ВАХ для тех же титановых нанопроводов, что и на рис. 2, приведены на рис. 3, $a$. Удивительно то, что обе ВАХ весьма похожи друг на друга, несмотря на радикально различное $R(T)$ поведение титановых электродов. Ранее уже сообщалось, что квантовые флуктуации приводят к слабому подавлению щели и размытию особенностей ВАХ в области щелевой особенности $|\mathrm{eV}| \sim \Delta(\mathrm{Ti})+\Delta(\mathrm{Al})[22-24]$. В частности, было показано, что „пролезание“ состояний в подщелевую область есть следствие гауссовых флуктуаций фазы параметра порядка, ведущих к возбуждению волн зарядовой плотности [19], с которыми взаимодействуют электроны проводимости, что приводит к перенормировке электронной плотности состояний [22-25]. Кроме очень похожих на стандартные туннельные БКШхарактеристики (правда, в нашем случае - с небольшой размытостью плотности состояний), все исследованные 

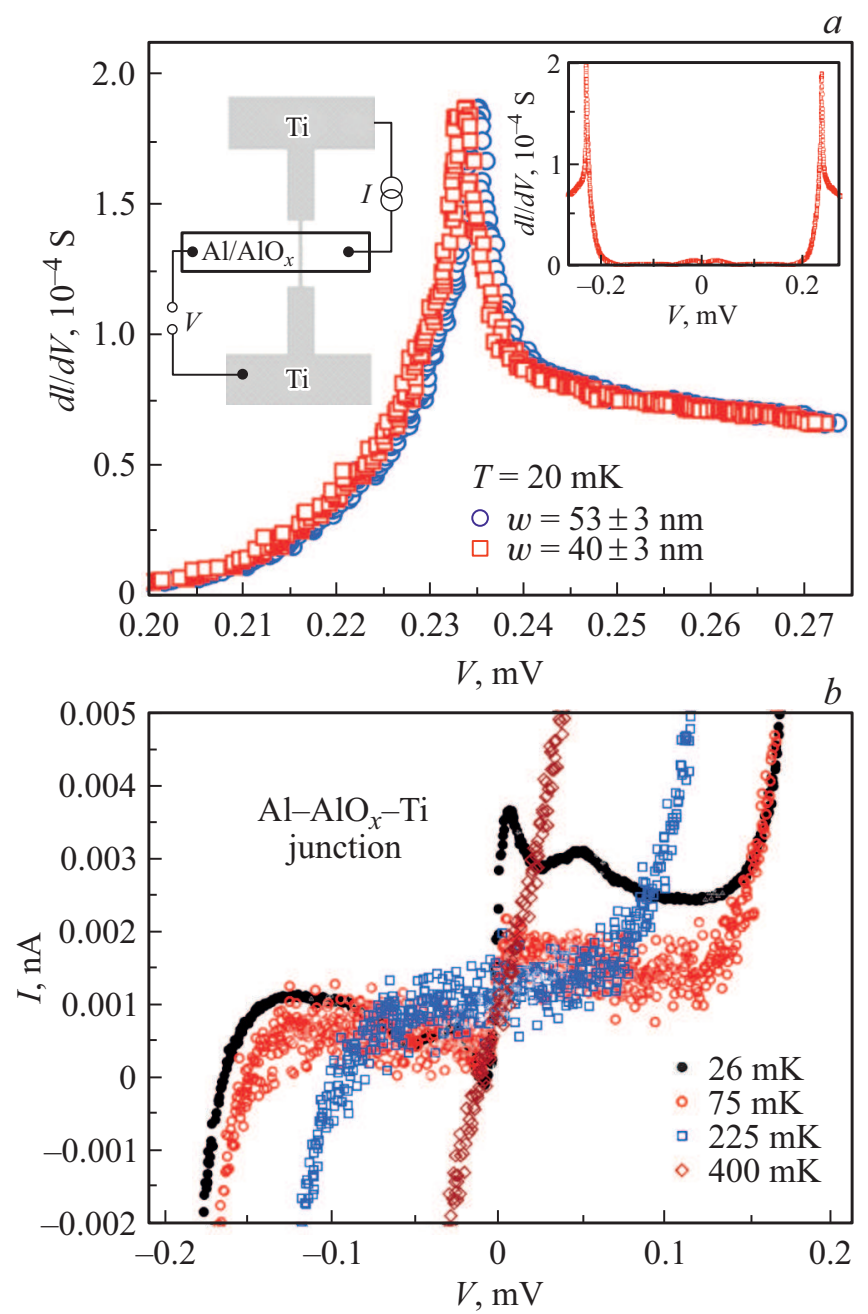

Рис. 3. а) Дифференциальные $d I / d V(V)$ характеристики двух туннельных контактов $\mathrm{Al}-\mathrm{AlO}_{x}-\mathrm{Ti}$ в области смещения порядка суммарной сверхпроводящей щели $\mathrm{eV} \sim \Delta(\mathrm{Ti})+\Delta(\mathrm{Al})$, образованных теми же самыми титановыми нанопроводами, что и на рис. 2. На левой вставке показана схема измерений. На правой вставке приведены те же самые зависимости в широком диапазоне смещений: в таком масштабе разница между двумя $d I / d V$ кривыми практически не заметна. $b)$ Вольт-амперная зависимость $I(V)$ в области малых смещений $|\mathrm{eV}| \ll \Delta(\mathrm{Ti})+\Delta(\mathrm{Al})$. Хорошо виден джозефсоновский ток, который сильно подавляется температурой и полностью исчезает выше критической температруры сверхпроводящего титана. Небольшое смещение экспериментальных точек по токовой оси связано с дрейфом нуля аналогового усилителя.

$\mathrm{Al}-\mathrm{AlO}_{x}-\mathrm{Ti}$ контакты демонстрируют также эффект Джозефсона (рис. 3,b). За счет присутствия квантовых флуктуаций в титановом электроде критический ток сильно подавлен по сравнению с величиной, следующей из соотношения Амбегаокара-Баратова, и исчезает при температурах выше критической. Из сказанного можно сделать вывод, что локальные атрибуты сверхпроводимости, определяющие форму туннельных зависимостей, слабо подавляются квантовыми флуктуациями параметра порядка.

\section{4. Заключение}

Экспериментально и теоретически было показано, что в квазиодномерных сверхпроводящих каналах из титана интегральные (эффективное сопротивление) и локальные (энергетическая щель) характеристики по-разному подавляются квантовыми флуктуациями параметра порядка. В достаточно тонких нанопроводах при самых низких температурах может наблюдаться конечное электрическое сопротивление, тогда как туннельные вольтамперные характеристики обнаруживают лишь слегка размытые щелевые особенности и конечный джозефсоновский ток. Такое состояние вещества можно рассматривать как сверхпроводящий изолятор, который возникает в результате слабой кулоновской блокады куперовских пар. Это явление универсально и, как показано в настоящей работе, наблюдается также в достаточно однородных сверхпроводящих каналах, не содержащих туннельных барьеров, что отражает фундаментальную общность свойств квазиодномерного сверхпроводника, в режиме квантовых флуктуаций, и джозефсоновского контакта малых размеров.

\section{Финансирование работы}

Работа выполнялась при поддержке проекта „Зеркальные лаборатории“ Национального исследовательского университета „Высшая школа экономики“ и Башкирского государственного педагогического университета им. М. Акмуллы.

\section{Конфликт интересов}

Авторы заявляют, что у них нет конфликта интересов.

\section{Список литературы}

[1] G.E. Moore. Electronics 38, 8, 1 (1965).

[2] O.V. Astafiev, L.B. Ioffe, S. Kafanov, Yu.A. Pashkin, K.Yu. Arutyunov, D. Shahar, O. Cohen, J.S. Tsai. Nature 484, 7394, 355 (2012).

[3] J.S. Lehtinen, K. Zakharov, K.Yu. Arutyunov. Phys. Rev. Lett. 109, 18, 187001 (2012).

[4] Z.M. Wang, J.S. Lehtinen, K.Yu. Arutyunov. Appl. Phys. Lett. 114, 24, 242601 (2019).

[5] K.Yu. Arutyunov, D. Golubev, A.D. Zaikin. Phys. Rep. 464, 1-2, 1 (2008).

[6] A.D. Zaikin, D.S. Golubev. Dissipative Quantum Mechanics of Nanostructures: Electron Transport, Fluctuations and Interactions. Jenny Stanford Publishing, Singapore (2019).

[7] A.D. Zaikin, D.S. Golubev, A. van Otterlo, G.T. Zimányi. Phys. Rev. Lett. 78, 8, 1552 (1997).

[8] A. Bezryadin, C.N. Lau, M. Tinkham. Nature 404, 6781, 971 (2000).

[9] M. Zgirski, K.-P. Riikonen, V. Touboltsev, K.Yu. Arutyunov. Phys. Rev. B 77, 5, 054508 (2008).

[10] J.S. Lehtinen, T. Sajavaara, K.Yu. Arutyunov, M.Yu. Presnjakov, A.L. Vasiliev. Phys. Rev. B 85, 9, 094508 (2012). 
[11] K.Yu. Arutyunov, T.T. Hongisto, J.S. Lehtinen, L.I. Leino, A.L. Vasiliev. Sci. Rep. 2, 293 (2012).

[12] A.G. Semenov, A.D. Zaikin. Phys. Rev. B 88, 5, 054505 (2013).

[13] M. Zgirski, K.-P. Riikonen, V. Touboltsev, K. Arutyunov. Nano Lett. 5, 6, 1029 (2005).

[14] J.S. Lehtinen, K.Yu. Arutyunov. Supercond. Sci. Technol. 25, 124007 (2012).

[15] K.Yu. Arutyunov, J.S. Lehtinen, T. Rantala. J. Supercond. Nov. Magn. 29, 569 (2016).

[16] V.V. Zavyalov, S.A. Chernyaev, K.V. Shein, A.G. Shukaleva, K.Yu. Arutyunov. J. Phys.: Conf. ser. 969, 012086 (2018).

[17] K.Yu. Arutyunov, J.S. Lehtinen, A.A. Radkevich, A.G. Semenov, A.D. Zaikin. To be published elsewhere (2021).

[18] D.S. Golubev, A.D. Zaikin. Phys. Rev. B 78, 14, 144502 (2008).

[19] J.E. Mooij, G. Schoen. Phys. Rev. Lett. 55, 1, 114 (1985).

[20] A. Radkevich, A.G. Semenov, A.D. Zaikin. Phys. Rev. B 100, 1, 014520 (2019).

[21] A. Radkevich, A.G. Semenov, A.D. Zaikin. J. Supercond. Nov. Magn. 33, 2335 (2020).

[22] K.Yu. Arutyunov, J.S. Lehtinen. Nanoscale Research Lett. 11, 1, 364 (2016).

[23] К.Ю. Арутюнов, J.S. Lehtinen, А.А. Радкевич, А.Г. Семенов, А.Д. Заикин. ФТТ 59, 11, 2092 (2017). Phys. Solid State 59, 11, 2110 (2017).

[24] K.Yu. Arutyunov, J.S. Lehtinen, A.A. Radkevich, A.G. Semenov, A.D. Zaikin. J. Magn. Magn. Mater. 459, 356 (2018).

[25] A. Radkevich, A.G. Semenov, A.D. Zaikin. Phys. Rev. B 96, 8,085435 (2017).

Редактор Е.В. Толстякова 